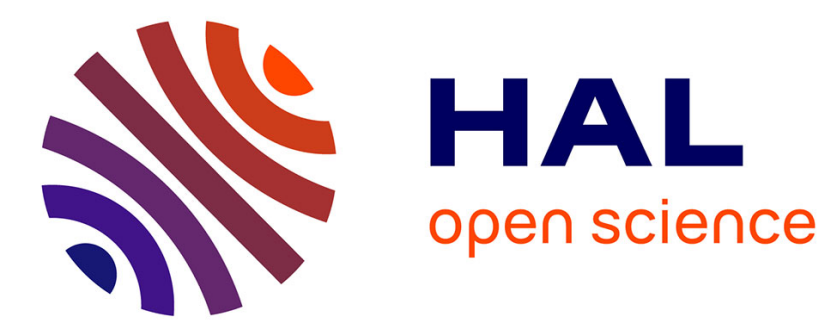

\title{
L'impact des TIC mobiles sur les activités des professionnels en entreprise
}

Charles-Henri Besseyre Des Horts, Henri Isaac

\section{To cite this version:}

Charles-Henri Besseyre Des Horts, Henri Isaac. L'impact des TIC mobiles sur les activités des professionnels en entreprise. 2006. halshs-00155192

\section{HAL Id: halshs-00155192 \\ https://shs.hal.science/halshs-00155192}

Preprint submitted on 15 Jun 2007

HAL is a multi-disciplinary open access archive for the deposit and dissemination of scientific research documents, whether they are published or not. The documents may come from teaching and research institutions in France or abroad, or from public or private research centers.
L'archive ouverte pluridisciplinaire HAL, est destinée au dépôt et à la diffusion de documents scientifiques de niveau recherche, publiés ou non, émanant des établissements d'enseignement et de recherche français ou étrangers, des laboratoires publics ou privés. 


\title{
L'IMPACT DES TECHNOLOGIES MOBILES D'INFORMATION ET DE COMMUNICATION SUR LES ACTIVITES DES PROFESSIONNELS EN ENTREPRISE : UNE ETUDE PERCEPTUELLE SUR LES SITUATIONS DE TRAVAIL *
}

\author{
Charles-Henri Besseyre des Horts \\ Professeur Associé \\ HEC Paris \\ 78350 Jouy en Josas Cedex \\ besseyre@hec.fr \\ Henri Isaac \\ Maître de Conférences \\ Dauphine Recherches en Management (CREPA), UMR CNRS 7088 \\ Université Paris Dauphine \\ 75775 Paris Cedex 16 \\ henri.isaac@dauphine.fr
}

* Cette recherche a été réalisée grâce au soutien de Toshiba France dans le cadre de la Chaire HEC-Toshiba « Mobilité \& Organisation » et conduite par une équipe de recherche conjointe HEC Paris-Dauphine Recherches en Management - CREPA (Université de Paris-Dauphine).

* This research was funded by Toshiba France as an endowment of the research Chair HECToshiba "Mobility \& Organization" and conducted by a joint research team HEC ParisDauphine Research in Management-CREPA (University of Paris-Dauphine). 


\title{
L'IMPACT DES TECHNOLOGIES MOBILES D'INFORMATION ET DE COMMUNICATION SUR LES ACTIVITES DES PROFESSIONNELS EN ENTREPRISE : UNE ETUDE PERCEPTUELLE SUR LES SITUATIONS DE TRAVAIL
}

\section{Résumé}

Il est largement reconnu que les technologies de l'information et de la communication (TIC) ont contribué à modifier les modes d'organisation depuis une dizaine d'années notamment avec l'usage généralisé des technologies mobiles : ordinateurs portables et téléphones mobiles. Des travaux récents mettent l'accent sur une transformation profonde des situations de travail permise par ces technologies dans le sens d'un développement de l'autonomie et de la responsabilité. Cependant, l'impact des technologies mobiles sur les activités des professionnels en entreprise semble plus complexe : d'une part, le fait de pouvoir être joint "n'importe où, n'importe quand" impose des exigences nouvelles en raison des modifications de l'espace-temps, d'autre part, le fait de pouvoir accéder partout à des informations cruciales pour l'exercice du travail donne à ces professionnels un pouvoir renforcé de contrôle sur leurs activités. À partir du cadre théorique proposé par le modèle "exigence-contrôle" (Karasek \& Theorell, 1990 ; Karasek, 1979), les résultats d'une étude exploratoire menée dans dix organisations françaises confirment l'hypothèse de conséquences paradoxales des technologies mobiles sur les situations perçues de travail par les professionnels en entreprise. Des enseignements pour les managers de ces professionnels et des pistes de recherches futures sont proposés en conclusion.

Mots clés : TIC mobile, situation de travail, professionnels, contrainte, autonomie.

\begin{abstract}
$\underline{\text { Abstract }}$
It is widely recognized that ICT have modified organizational structures and processes during the last decade, notably with the general usage of laptop computers and mobile phones. A number of research works have pointed out the deep transformation of work situations allowed by these technologies with the development of more autonomy and responsibility. However, in the case of mobile technologies, the impact of these technologies on professionals' work situations appears to be far more paradoxical: on the one hand, being connected "anywhere, anytime" induces new expectations because of the abolishment of time and space and, on the other hand, being able to access everywhere crucial information to complete work gives these professionals a reinforced control power upon their activities. Using the theoretical framework suggested by the "demand-control" model (Karasek \& Theorell, 1990; Karasek, 1979), results of an exploratory study conducted in 10 French organizations confirm this hypothesis of paradoxical consequences of mobile technologies on work situations by within firm professionals. Learnings for professionals' managers and future research orientations are proposed as a conclusion.
\end{abstract}

$\underline{\text { Key words: }}$ Mobile technology, work situation, professionals, constraint, autonomy. 


\section{L'IMPACT DES TECHNOLOGIES MOBILES D'INFORMATION ET DE COMMUNICATION SUR LES ACTIVITES DES PROFESSIONNELS EN ENTREPRISE : UNE ETUDE PERCEPTUELLE SUR LES SITUATIONS DE TRAVAIL}

\section{Introduction}

Les technologies de l'information et de la communication (TIC) ont largement contribué à modifier les structures et processus organisationnels depuis une dizaine d'années en raison notamment de la croissance exponentielle de l'usage des technologies réseaux et Internet en particulier. La caractéristique principale de l'évolution de ces technologies est la généralisation de l'usage des outils mobiles de communication comme le sont les ordinateurs portables et téléphones mobiles connectés à Internet par les réseaux sans fil actuellement disponibles (Wi-Fi,3G...). L'usage de ces outils mobiles transforme profondément les rapports traditionnels au temps et à l'espace qu'entretiennent les individus dans le contexte du travail et en dehors du travail (Chen \& Nath, 2005 ; Davis, 2002). Les technologies mobiles dotent les individus de capacités d'ubiquité en ce sens que ces derniers peuvent exercer leurs activités professionnelles potentiellement n'importe quand, n'importe où, voire dans des contextes inhabituels (Leclercq \& Isaac, 2005 ; Lyytinen \& Yoo, 2002a) y compris dans des situations de travail à distance (Cocula \& Fredy-Planchot, 2003).

Sur le plan des conséquences pour les individus, les avantages procurés par les technologies mobiles sont souvent mis en avant pour justifier l'adoption et le déploiement de ces outils dans le contexte du travail : une amélioration des capacités de communication, de coordination et de collaboration ; une possibilité renforcée d'accès aux décideurs, une meilleure réactivité et une plus grande autonomie dans les décisions. Mais ces avantages sont susceptibles d'être obérés par des inconvénients décrits comme potentiellement liés à l'usage de ces technologies : l'absence de frontière entre travail non travail, la discontinuité des activités, le culte de l'urgence, l'absence de réflexion dans les décisions, la surcharge informationnelle (Isaac, Kalika, Campoy 2006), le contrôle renforcé des activités (Jarvenpaa \& Lang, 2005 ; Gribbins et al, 2003 ; Davis, 2002).

Dans le cadre de cette révolution technologique, les professionnels, dont la nature particulière des activités et responsabilités a fait l'objet de très nombreux travaux (e.g. Swailes, 2003 ; Gadrey, 2000 ; Morrow \& Goetz, 1988 ; Hall, 1968), sont directement concernés dans la mesure où les technologies mobiles sont susceptibles de renforcer les exigences d'autres acteurs (supérieurs hiérarchiques, collègues, clients...) qui peuvent les joindre n'importe où, n'importe quand, mais aussi simultanément de développer le contrôle qu'ils ont dans l'exercice de leurs activités. Cette contribution se propose d'examiner théoriquement et empiriquement cette conséquence paradoxale de l'usage des technologies mobiles dans les situations de travail telles qu'elles sont perçues par les. Après avoir présenté les caractéristiques des technologies mobiles et leurs conséquences potentiellement paradoxales, la situation particulière des professionnels en entreprise sera examinée avant de décrire le modèle "exigence-contrôle" (demand-control model) (Claessens et al, 2004 ; Van Yperen \& Hagedoorn, 2003 ; Karasek \& Theroell, 1990 ; Johnson \& Hall, 1988). Les résultats d'une recherche empirique auprès de dix entreprises françaises confirment l'existence de conséquences paradoxales, en termes d'exigence et de contrôle des technologies mobiles sur les situations de travail des professionnels en entreprise. Des enseignements pour les managers de ces professionnels et des pistes de recherches futures sont proposés en conclusion. 


\section{Les technologies mobiles et leurs effets paradoxaux}

\subsection{Les technologies mobiles en entreprise}

Les technologies mobiles font ici référence à un ensemble de technologies (outils, réseaux, logiciels...) dont les usages sont différents mais souvent complémentaires et qui ont même tendance à se rapprocher de plus en plus dans le cadre d'une convergence de ces technologies (Isaac, 2004, Lyytinen \& Yoo, 2002b).

Parmi les outils, le téléphone mobile est l'outil le plus répandu en entreprise et dont l'usage est aussi bien professionnel que personnel. L'agenda électronique personnel (ou PDA) a tendance à régresser au profit du téléphone intelligent (smartphone) combinant simultanément les usages du téléphone mobile et d'un agenda électronique. L'ordinateur portable, occupe également une place importante dans les outils à la disposition des salariés nomades. D'autres outils peuvent être cités comme le tabletPC qui reste confidentiel et la clé USB qui représente un outil de mobilité largement répandu en entreprise. Mais ces outils ne seraient pas des outils de mobilité s'il n'y avait pas d'infrastructures de réseaux sans fil couvrant des zones plus ou moins étendues : par exemple, les réseaux $3 \mathrm{G}$ et les réseaux Wi-fi qui peuvent être utilisés, selon les possibilités techniques, par la plupart des outils mobiles disponibles.

Dans l'entreprise, l'usage des technologies mobiles est variable selon les catégories d'employés (Isaac, 2006). Les collaborateurs traditionnellement les plus mobiles (commerciaux, techniciens de maintenance, consultants) et les managers " nomades" sont ceux qui sont prioritairement concernés par le déploiement des outils mobiles (Isaac, Leclercq \& Besseyre des Horts, 2006 ; Leclercq \& Isaac, 2005 ; Isaac, 2004). Parmi ces deux dernières catégories, la première (commerciaux, techniciens de maintenance, consultants) regroupe pour une large part les professionnels en entreprise tels qu'ils seront définis plus loin. Pour les autres catégories, l'utilisation des technologies mobiles est souvent liée à des choix personnels.

\subsection{Les effets paradoxaux des technologies mobiles}

Comme le souligne Varshney (2003), les technologies mobiles sont un moyen d'introduire dans les organisations une forme nouvelle de "flexibilité, en termes de lieux et de temps" et, en ce sens, sont porteuses de nombreuses promesses pour les entreprises (Leung \& Antypas, 2001). L'ubiquité ainsi permise signifie que les salariés peuvent se connecter au système d'information de leur entreprise et être joints par celle-ci à tout moment et en tout lieu (Robey et al., 2004). Les avantages à en retirer sont nombreux, au premier rang desquels l'accroissement de la productivité individuelle grâce à la réduction des exigences spatiales et temporelles dans la réalisation du travail, l'accroissement de la flexibilité, la diminution des coûts de coordination, l'amélioration de la communication et de l'échange de connaissances. En outre, les technologies mobiles facilitent l'immédiateté de l'accès à l'information, la hausse de la performance dans la prise de décision, et, ainsi, l'accroissement de la réactivité face aux clients (Gribbins, Shaw, Gebbauer, 2003 ; Davis, 2002 ; Churchill \& Munro, 2001). Pour la catégorie spécifique des professionnels utilisant des outils mobiles de communication, Kakihara et Sorensen (2004) montrent que ces technologies leur permettent de mieux exercer leurs activités en diffusant notamment leur expertise auprès de leurs clients internes ou externes.

Cependant, il semblerait que les managers prennent progressivement conscience des nouvelles exigences dues à l'utilisation des technologies mobiles et d'une nouvelle forme d'astreinte qui s'impose progressivement à eux (Management, 2006 ; Isaac, 2004 ; Davis, 2002). 
Au sein de l'entreprise, le téléphone mobile apparaît en effet comme un instrument au service de l'indépendance et de la mobilité des salariés. Il n'en est pas moins également le symbole d'un maintien des "chaînes hiérarchiques" au-delà même des frontières de l'entreprise. L'utilisation par les salariés des technologies mobiles permet potentiellement aux entreprises, au travers d'une "traçabilité digitale" (Robey et al., 2004), d'exercer sur eux un contrôle et une surveillance continus, en dehors de l'espace de travail, ce qui ne va pas sans provoquer un certain stress (Churchill \& Munro, 2001) et accroître les risques d'empiètement sur la vie privée (Cousins et Robey, 2005 ; Genin \& Besseyre des Horts, 2004; Isaac \& Kalika, 2001; Wiberg \& Grönlund, 1999)

L'utilisation des technologies mobiles par les salariés pose également des problèmes de fragmentation et d'interruptions dans le travail "favorisant la distraction plutôt que le temps de la réflexion" ( Davis, 2002 ; Ettighoffer, 2001). Les individus peuvent se sentir oppressés par l'émergence d'une véritable "culture de la vitesse et de l'instantanéité" qui les oblige à prendre des décisions dans l'urgence, ou dans des contextes inadaptés à la prise de décision, ce qui peut s'avérer finalement contre-productif (Aubert, 2003).

Au regard des recherches sur l'impact des technologies mobiles, il apparait clairement que ces dernières sont susceptibles de générer des effets ambivalents voire paradoxaux, et de provoquer des conséquences "ironiques et perverses" (Arnold, 2003). Cousins et Robey (2005) mettent ainsi l'accent sur l'opposition entre les bénéfices attendus et les conséquences sociales inattendues des environnements informatiques nomades. De leur côté, Jarvenpaa \& Lang (2005) présentent huit conséquences paradoxales des TIC mobiles à partir d'une étude des perceptions de 222 utilisateurs d'outils mobiles (essentiellement des téléphones mobiles et des smartphones): liberté / esclavage, indépendance / dépendance, satisfaction des besoins / création des besoins, compétence / incompétence, planification / improvisation, engagement / désengagement, public/privé, illusion / désillusion.

Bien que cette étude s'intéresse à une population plus large que celle des salariés, plusieurs paradoxes mis en évidence par Jarvenpaa et Lang (2005) peuvent être rapprochés des problématiques de l'exigence (contrainte) dans les situations de travail et du contrôle de l'activité (latitude de décision). Ainsi les paradoxes liberté/esclavage, indépendance/dépendance, planification/improvisation et engagement/désengagement peuvent représenter des éléments constitutifs du contrôle (latitude de décision) et de l'exigence (contrainte). Une situation de travail caractérisée par l'exigence, voire par une forme de néotaylorisme, pourrait être décrite par une situation d'esclavage (même si ce terme est excessif), de dépendance, de planification, et de désengagement. Une situation de travail, à l'inverse, caractérisée par la latitude de décision pourrait en effet être décrite comme une situation de liberté, d'indépendance, d'improvisation et en définitive d'engagement.

Ces conséquences paradoxales concernent bien évidemment les professionnels que sont, par exemple, les commerciaux et techniciens de terrain ou les consultants. Après avoir rappelé les caractéristiques principales des professionnels en entreprise, la question de l'exigence et du contrôle sera présentée à partir du modèle "exigence-contrôle" élaboré par Karasek (1979) qui a été fondateur d'un courant de recherche parmi les plus influents dans le champ de la psychologie sociale depuis plus de vingt ans (Van der Doef \& Maes, 1999).

\section{Les professionnels en entreprise : une catégorie particulière de collaborateurs mobiles}

La catégorie des professionnels se distingue des autres catégories de travailleurs par des caractéristiques dont les principales ont été proposées il y a plusieurs décennies dans des 
travaux fondateurs en sociologie (e.g. Hall, 1968 ; Wilensky, 1964). Selon ces travaux, le développement d'une profession suppose la réalisation des conditions suivantes : 1) la création d'une activité professionnelle à plein temps satisfaisant un besoin social reconnu, 2) le développement d'un parcours de formation propre à la profession pour la création et la diffusion de la connaissance, 3) la création d'une association professionnelle dont les membres définissent clairement la nature des activités professionnelles et mettent en place éventuellement un dispositif formel de reconnaissance (accréditation), 4) l'élaboration d'un code éthique de la profession réglementant les relations avec les différentes parties prenantes. Comme le souligne Hall (1968), une caractéristique importante des professionnels est leur autonomie dans l'exercice de leurs activités : on attend des professionnels qu'ils utilisent leur expertise et leur expérience et que seuls d'autres professionnels soient compétents pour les évaluer.

Hall (1968) montre également qu'il y a un lien étroit entre les attitudes et les comportements des professionnels. Sur le plan des attitudes, en effet, les professionnels 1) se reconnaissent comme membre d'une communauté formalisée, le cas échéant, par l'existence d'une association professionnelle, 2) définissent leur légitimité par l'activité professionnelle qu'ils exercent pour répondre à un besoin social, 3) croient dans l'auto-régulation de la profession, ce qui implique que d'autres professionnels peuvent être amenés à juger la qualité du travail réalisé, 4) s'impliquent fortement dans des contextes de travail où ils exercent leurs activités et 5) sont autonomes dans la mesure où ils sont capables de prendre leurs propres décisions sans en référer au jugement d'autres acteurs (supérieurs hiérarchiques, collègues, clients...).

Plus récemment, Lui et al (2003) identifient les cinq conditions suivantes qui décrivent les dimensions essentielles du professionnalisme : 1) l'acquisition de connaissances spécifiques à la profession et qui doivent être développées, transmises et utilisées dans l'exercice des activités, 2) l'action indépendante en prenant des décisions fondées sur l'expérience et le jugement personnel, 3) l'acceptation d'un statut qui distingue les professionnels par leur identité propre, 4) la relation d'aide dans laquelle les prestations des professionnels peuvent aller au-delà de la simple activité négociée ou prescrite 5) la démonstration d'un engagement professionnel dans des processus de socialisation largement basés sur des valeurs éthiques

Enfin, sur le plan des facteurs qui motivent les professionnels, Katz (2005) montre que ces derniers, notamment dans la $R \& D$, se distinguent des autres par le fait qu'ils cherchent à apprendre et développer des nouvelles compétences et capacités, à devenir des membres contribuant au développement de la profession, à travailler sur des projets qui les stimulent au sein de leur profession, à renforcer leur autonomie opérationnelle, et à être évalués avec des données objectives.

Tout en se différenciant des autres catégories de travailleurs, les professionnels n'en constituent pas pour autant une catégorie homogène. Le terme de "professionnel" fait souvent en effet référence à une catégorie d'acteurs qui se reconnaissent au sein d'une profession clairement identifiée (médecins, architectes, avocats, consultants...) et qui exercent leurs activités au sein d'organisations dans lesquelles ils sont en position d'indépendance : sur le plan juridique, le contrat qui les lie généralement à ces organisations est de nature commerciale. À l'inverse, les professionnels en entreprise sont liés par un contrat de travail caractérisé par la relation de subordination à leur employeur mais ils conservent cependant l'essentiel des autres caractéristiques telles quelles ont été décrites plus haut.

Compte-tenu de l'autonomie d'actions qui les caractérise, les professionnels en entreprise sont beaucoup plus susceptibles que d'autres de devenir des collaborateurs mobiles dans la mesure où ils mettent au service de l'entreprise leurs compétences et expérience partout où la demande interne ou externe est exprimée. Ainsi, par exemple, un consultant interne en 
organisation sera-t-il amené à intervenir partout où se posent des questions de réorganisation et de changement des processus. De ce point de vue, ils sont beaucoup plus "cosmopolites" que "locaux" pour reprendre la célèbre distinction de Gouldner (1958). Cette dimension cosmopolite a été renforcée depuis quelques années avec le développement d'Internet qui accélère les processus de socialisation avec la création notamment des communautés de pratiques constituant des univers virtuels dans lesquels les professionnels en entreprise peuvent renforcer la profession sans jamais se rencontrer physiquement (Harshman et al, 2005). Les technologies mobiles ont également un impact important sur les conditions d'exercice de leurs activités par les professionnels : Sorensen et Gobson (2005) montrent, par exemple, que ces technologies sont perçues par les professionnels comme étant indispensables pour l'exercice de leurs activités sans pour autant être une condition suffisante au développement du professionnalisme. Les technologies mobiles permettent, en effet, aux professionnels interrogés d'avoir plus grand contrôle de leurs activités, c'est-à-dire une plus grande latitude dans leurs décisions, mais sont également perçues par eux comme renforçant les contraintes de leur situation de travail dans la mesure où ils peuvent être joints n'importe où et n'importe quand grâce à ces outils mobiles.

Cette conséquence paradoxale, simultanément une plus grande latitude dans les décisions et plus de contraintes dans la situation de travail, de l'usage des technologies mobiles par les professionnels en entreprise est désormais analysée à l'aide du modèle exigence-contrôle (Karasek, 1979).

\section{Exigence et contrôle dans la perception des situations de travail des professionnels en entreprise : un cadre théorique}

La question de l'exigence et du contrôle est une question récurrente dans les réflexions sur l'organisation et le travail comme le soulignent les synthèses sur les grands courants en théories de l'organisation (Rojot, 2003 ; Livian, 1995, Aubert et al, 1991). En s'appuyant sur ces analyses, il est possible de souligner que les théories rationnelles sont fortement dominées par l'impératif de l'exigence dans la mesure où le travail peut être scientifiquement organisé selon les principes de l'organisation taylorienne ou structuré en activités liées à la prise en compte des contraintes de l'environnement comme le proposent les tenants de l'école de la contingence structurelle. Inversement, les courants relevant de l'approche sociale (Aubert et al, 1991), privilégient nettement la dimension du contrôle de la situation de travail (ou latitude de décision) si l'on se réfère, par exemple, aux travaux des grands théoriciens de la motivation qui ont tous en commun une conviction partagée : une plus grande maîtrise de la situation de travail est à la source d'une plus forte motivation des individus. Plus récemment, les tenants du management participatif et de "l'empowerment" ont défendu l'idée selon laquelle il était nécessaire de développer l'autonomie et la responsabilité au travail pour mobiliser les individus; cette dernière idée est d'autant plus importante, que nos sociétés s'orientent de plus en plus vers des sociétés de la connaissance dans laquelle les individus se trouvent dans des situations de travail où l'exigence cède progressivement du terrain à l'autonomie.

Cette question est au coeur du modèle "exigence-contrôle" (demand-control model) élaboré par Karasek (1979) qui a mis l'accent très tôt sur le fait, que les recherches sur les effets psychosociaux des environnements de travail s'intéressaient soit aux facteurs d'exigence soit aux facteurs de contrôle mais rarement aux deux simultanément. L'intérêt principal du modèle de Karasek (1979) est de proposer un cadre théorique intégrant simultanément les facteurs de l'exigence et du contrôle. 
Dans son article fondateur, Karasek (1979) justifie la nécessité de prendre en compte simultanément les facteurs de l'exigence de la situation travail, qu'il regroupe sous le terme générique de "demand" et les facteurs qui contribuent au développement de contrôle de la situation de travail, qu'il décrit comme "la latitude de décision au travail" (job decision latitude) mais que la plupart des recherches, qui se sont inspirées du modèle de Karasek (1979), ont synthétisé sur le terme de "control"1.

Comme le soulignent Van Yperen \& Hagedoorn (2003), l'idée principale de ce modèle est de montrer qu'une exigence forte (contrainte) de la situation de travail associée à une faible autonomie crée la situation de travail la plus stressante puisque l'individu ne peut pas répondre à cette exigence (contrainte) dans la mesure où il n'a pas le contrôle de sa situation de travail. Dans leur revue des recherches empiriques menées pendant 20 ans à partir des travaux de Karasek (1979), Van der Doef \& Maes (1999) présentent le modèle "demand-control" en soulignant, d'une part, que les facteurs d'exigence (ou "demand" dans le modèle) font référence à des facteurs comme la pression du temps et conflit de rôle et, d'autre part, que les facteurs de contrôle correspondent à la poly compétence et l'autorité de décision. Ils représentent l'interaction entre ces facteurs sous la forme de la figure suivante (adaptée de Karasek, 1979) :

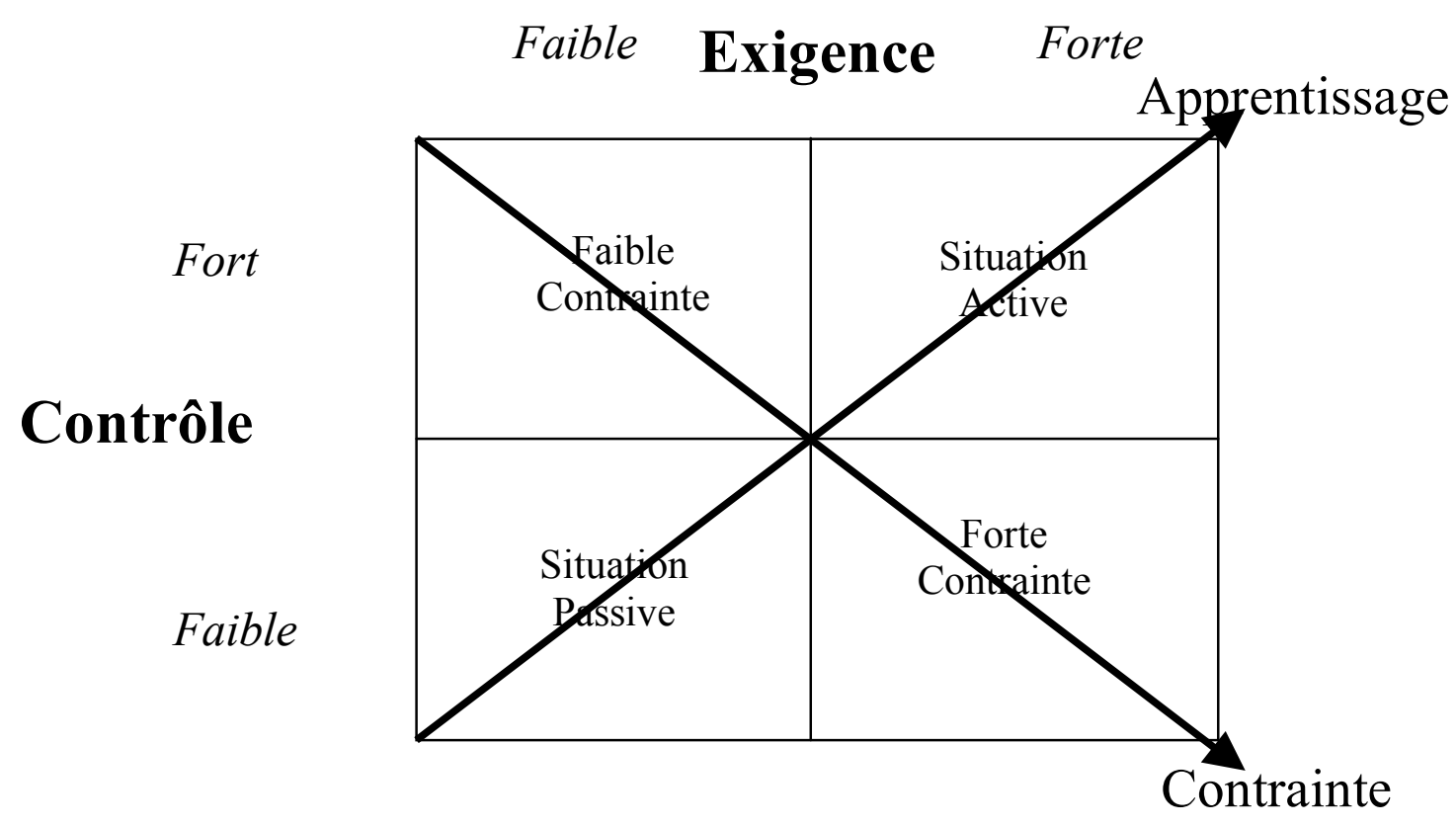

Figure 2: Le modèle Exigence-Contrôle (Van der Doef \& Maes, 1999, p.88)

Comme le montre cette figure, les deux facteurs de l'exigence et du contrôle sont en interaction dans la mesure où quatre situations de travail sont théoriquement possibles : celle, déjà citée plus haut, la plus contraignante où l'exigence est forte et le contrôle est faible ; à l'inverse, la situation peu contraignante où l'exigence est faible et le contrôle est fort. Sur l'autre diagonale, le modèle met l'accent sur la dimension de l'apprentissage puisque la situation où l'exigence et le contrôle sont faibles est qualifiée de situation passive alors que la situation inverse où l'exigence et le contrôle sont forts est qualifiée de situation active.

\footnotetext{
${ }^{1}$ Contrôle ici compris comme la capacité d'agir sur sa situation de travail, c'est-à-dire être autonome : voir Van der Doef \& Maes (1999)
} 
Certains auteurs (Van Yperen \& Hagedoorn, 2003 ; Van der Doef \& Maes, 1999) ont mis en évidence le fait que cette interaction pouvait être comprise comme un effet de médiation de l'exigence par le contrôle, en d'autres termes les effets négatifs d'une situation de travail très exigeante (i.e à fortes contraintes) seront d'autant plus faibles de le contrôle (i.e. la capacité à contrôler sa situation de travail) sera forte.

Ce modèle est utilisé comme cadre théorique pour analyser les conséquences paradoxales de l'usage des technologies mobiles sur la perception des situations de travail par les professionnels en entreprise dans la mesure où ces technologies génèrent des situations avec des niveaux d'exigence plus ou moins forts est une latitude de décision plus ou moins réelle. L'application du modèle conduit à la formulation des trois propositions :

Proposition $1: L^{\prime}$ acceptation (ou le rejet) des technologies mobiles par les professionnels en entreprise est liée à leur perception des risques perçus en termes de perte de contrôle des activités (latitude de décision), en d'autres termes de leur autonomie.

Proposition 2 : Les technologies mobiles sont perçues, par les professionnels en entreprise, comme développant leur capacité de contrôle des activités (latitude de décision) qui, combinée à une exigence plus forte dans la situation de travail, développe leurs capacités d'apprentissage.

Proposition 3: Les technologies mobiles sont perçues, par les professionnels en entreprise, comme renforçant leur niveau de contrainte contraintes en raison, simultanément, d'une augmentation de l'exigence de la situation de travail et d'une diminution de leur capacité de contrôle des activités (latitude de décision).

\section{Méthodologie}

\subsection{Design de la recherche}

Les données ont été collectées dans le cadre d'une recherche plus large sur les technologies mobiles. Compte tenu du faible nombre de travaux de recherches sur ce thème, une approche exploratoire a été choisie en adoptant une démarche de recherche qualitative reposant sur des entretiens semi-structurés.

Les personnes interrogées sont des responsables opérationnels, commerciaux et autres collaborateurs terrains (techniciens de maintenance, agent de saisie de données terrain...). La démarche exploratoire est construite à partir d'une double perspective : déductive et inductive. La perspective déductive s'est appuyée sur l'analyse des travaux identifiés dans la littérature sur les conséquences humaines et organisationnelles des technologies mobiles de communication, permettant alors de construire, en partie, un guide d'entretiens semi-structurés (cf. annexe). La perspective inductive a résulté de l'analyse des entretiens et des thèmes émergents. Chaque entretien a duré entre de $45 \mathrm{mn}$ et une heure trente.

Compte tenu du fait que la recherche, examine également les questions de l'adoption, du développement, et des conséquences de l'usage des technologies, seule une partie du guide d'entretien a été utilisée pour recueillir les perceptions sur les situations de travail (notamment en termes de perceptions des exigences de la situation de travail et de contrôle des activités perçues).

\subsection{Recueil et analyse des données}

La recherche a été menée dans dix grandes organisations françaises, représentant les secteurs variés (industriels, professions de services et de la santé). Sur l'ensemble des entretiens réalisés par l'équipe de recherche lors du premier semestre 2005, les données de 39 entretiens 
ont été retenues dans la mesure où les répondants correspondaient aux critères définissant les professionnels en entreprise (cf. tableau 1). Les entretiens ont été enregistrés et retranscrits., puis codés à l'aide d'une procédure de codage utilisant le logiciel Nvivo afin d'identifier les différences de perceptions sur les situations de travail et d'en repérer les dimensions liées à l'exigence et au contrôle.

\begin{tabular}{|c|c|c|c|}
\hline & $\begin{array}{c}\text { Responsables } \\
\text { opérationnels }\end{array}$ & $\begin{array}{c}\text { Commerciaux } \\
\text { Collaborateurs } \\
\text { de terrain }\end{array}$ & Total \\
\hline Entreprise 1 (E1) & 1 & 4 & $\mathbf{5}$ \\
\hline Entreprise 2 (E2) & 0 & 4 & $\mathbf{4}$ \\
\hline Entreprise 3 (E3) & 0 & 2 & $\mathbf{2}$ \\
\hline Entreprise 4 (E4) & 1 & 2 & $\mathbf{3}$ \\
\hline Entreprise 5 (E5) & 1 & 3 & $\mathbf{4}$ \\
\hline Entreprise 6 (E6) & 0 & 3 & $\mathbf{3}$ \\
\hline Entreprise 7 (E7) & 0 & 4 & $\mathbf{4}$ \\
\hline Entreprise 8 (E8) & 0 & 5 & $\mathbf{5}$ \\
\hline Entreprise 9 (E9) & 1 & 4 & $\mathbf{5}$ \\
\hline Entreprise 10 (E10 & 0 & 4 & $\mathbf{4}$ \\
\hline Total & $\mathbf{4}$ & $\mathbf{3 5}$ & $\mathbf{3 9}$ \\
\hline
\end{tabular}

$\underline{\text { TTableau } 1}$ : Echantillon des répondants

\section{Résultats et Discussion}

\subsection{Adoption des technologies mobiles par les professionnels en entreprise: acceptation et résistance}

L'analyse des entretiens s'intéresse dans un premier temps au degré d'acceptation des outils mobiles par les professionnels en entreprise. Les répondants les plus enthousiastes quant à l'adoption de ces technologies font souvent référence à une culture en évolution qui privilégie le développement de ces outils en faveur d'un plus grand professionnalisme :

" Mais ça fait partie de la culture de notre entreprise d'utiliser les nouvelles technologies, notamment dans le cadre de la représentation commerciale sur le terrain. D'être toujours joignable pour le service client, c'est très important pour nous, ça veut dire téléphone, email, call center... En externe la notion d'être joignable pour nos clients, c'est très important. A l'époque de la non communication mobile, le mode de communication le plus courant c'était le courrier, ce qui n'est pas génial au niveau du contact avec le client. s" (Resp. opérationnel, E2)

"C'est venu naturellement, avant non, mais c'est venu naturellement, le téléphone portable, l'informatique ce n'est que... Avant on se doutait que ça amènerait des aspects intéressants, des aspects constructifs. Ca allait dans le sens de la nouveauté, comme les voitures. La technologie évolue sur les voitures et ça va 
dans le sens du client en terme de sécurité en termes de confort, en termes de conduite, en termes d'agrément de conduite donc, moi je dirais que la technologie n'est que du plus pour tout le monde".(Commercial, E5)

"On ne se pose même plus la question de savoir si c'est des objets utiles. Ils sont rentrés dans nos habitudes. Comment on faisait avant?" (Commercial, E8)

L'acceptation des outils mobiles est également expliquée par certains répondants comme satisfaisant aux exigences de métiers de plus en plus professionnels. En d'autres termes, ces technologies mobiles s'imposent d'elles-mêmes sans qu'il soit possible de les remettre en cause avec le risque de générer les situations de forte contrainte comme le montre le modèle "exigence-autonomie" (Karasek, 1979) :

" On est obligé de s'en servir. Quand on dit, qu'on a envie de déconnecter etc. le week-end c'est comme ça marche. Ca fait partie de métier. S'il y a des technologies pour nous aider, autant s'en servir." .(Resp opérationnel, E4)

J'ai tout se suite vu tous les avantages que ça donnait. On gagne du temps, on ne perd pas un papier, ni un dossier à moins d'être une tâche complète on arrive à suivre nos dossiers. Les formations c'est deux fois par an. Pour le téléphone, j'en avais un depuis longtemps. c'est vraiment pas compliqué. J'y ai vu l'intérêt pour la profession. On ne se perd plus dans les banlieues quand on débute sur un terrain on peut téléphoner et on vous guide...on a un GPS aussi." (Resp. opérationnel, E8)

"Je pense qu'aujourd'hui tout le monde l'apprécie complètement. De toute façon, aujourd'hui le papier.. Et puis aujourd'hui même nous, ne serait-ce que pour notre activité, on vend des prestations à nos clients, qui sont des prestations sur le Web, donc on leur vend aussi la suppression de papier. zéro papier. Si nous derrière, on travaille différemment... Il doit $y$ avoir une certaine cohérence..."(Commercial E10)

A l'inverse des partisans des technologies mobiles, les répondants qui se sont montrés beaucoup plus réservés à propos de l'adoption et de l'utilisation de ces outils mobiles mettent en avant également des raisons de traditions et de culture, ce qui traduit une méfiance par rapport à l'évolution des nouveaux comportements professionnels attendus.

"Mais nous, un peu plus âgé, on n'a jamais travaillé sur ordinateur, on ne connaissait pas, on ne savait pas s'en servir, et on a eu... On avait plutôt un peu peur de ne pas y arriver, mais bon forcément on se débrouille, on arrive à se débrouiller" (Collaborateur terrain, E1)

"On a des commerciaux qui ne sont pas âgés qui ont 46-50 ans et qui sont hermétiques à l'outil informatique. Ce qui est intéressant c'est qu'en général lorsqu'ils sont hermétiques à cet outil, ils sont hermétiques à tout. C'est-à-dire à toute forme nouvelle d'activité, c'est plus un problème de personne que d'outil..." (Resp. opérationnel, E2)

"Sinon, je n'ai rien d'autre et je fais partie de ceux qui reculent pour avoir un organiseur. Pour l'instant, je l'ai même refusé parce que ça tombe en panne sans arrêt. Bon peut être que dans notre entreprise on n'a pas mis les moyens dans la qualité, mais je me rends compte quand on est en réunion et qu'on veut prendre un rendez-vous que je vais beaucoup plus vite avec mon petit agenda. D'autant plus que c'est vrai que ça peut être utile car si on est connecté à l'ordinateur, les 
secrétaires savent où on est, mais on a d'autres moyens pour ça..." (Collaborateur terrain, E7)

En dehors de raisons de culture et de traditions, c'est la peur de l'inconnu qui est avancée par des répondants qui expriment souvent ici des craintes de pertes du contrôle de leurs activités, c'est-à-dire de leur autonomie, une caractéristique essentielle de leur rôle en tant que professionnels en entreprise. Pour reprendre le modèle "exigence-contrôle" (Karasek, 1979), ces répondants craignent que les technologies mobiles transforment leur situation de travail d'une situation active à une situation de forte contrainte :

"Quand vous le dotez d'outils, sa première réaction c'est de penser qu'il va perdre du temps. Il est organisé pour un certain nombre de tâches, quand on lui greffe une tâche qui a vocation à lui faire gagner du temps, ce n'est pas ce qu'il voit en premier. Il voit qu'il va falloir remplir des tableaux dont il n'a pas l'habitude.." (Resp opérationnel, E2)

" J'ai un collègue qui ne cherche même pas. Il a 35 ans de maison, il vient de voir, il me fait faire le travail, il ne cherche même pas, des fois je lui montre, il ne regarde pas. Des fois chez lui il n'arrive pas, il m'appelle. C'est vraiment une minorité."(Commercial, E2)

"... au début j'ai eu aussi l'impression que je perdais quelque chose comme si l'outil allait me prendre ce que j'aime dans ce métier le contact humain direct. Et puis aussi une perte du savoir qui était dans ma tête. Jamais un PC ne saura comment parler d'un produit à Monsieur Dupont comme je sais le faire. Un coup de téléphone peut régler un soucis ou une prise de $R V$ mais n'arrivera pas à convaincre untel d'une mise en rayon de tel produit" (Resp. opérationnel, E8)

En définitive, l'acceptation d'utiliser les outils mobiles dépend largement des avantages et des risques perçus par les professionnels notamment en termes de contrôle de leurs activités. Si certains perçoivent dans l'utilisation des technologies mobiles une occasion pour développer leur professionnalisme en leur donnant plus de latitude dans leurs décisions, d'autres les considèrent comme une menace sérieuse à l'encontre de leur autonomie qui constitue l'une des caractéristiques les plus importantes à leurs yeux de leurs métiers en tant que professionnels en entreprise. Ces résultats tendent à valider la première proposition de recherche.

\subsection{Technologies mobiles et situations de travail : des opportunités d'apprentissage pour les professionnels en entreprise}

Lorsqu'on examine la littérature disponible sur l'impact humain et organisationnel des technologies mobiles, les avantages surpassent largement les inconvénients. Pour les individus, les avantages les plus cités sont les suivants : une amélioration des capacités de communication, de coordination et de collaboration; une réduction des exigences de temps et d'espace dans l'exercice des activités ; une possibilité renforcée d'accès aux décideurs, une meilleure réactivité et une plus grande autonomie dans les décisions. Dans la perspective du cadre théorique présenté plus haut (Karasek \& Theroell, 1990 ; Karasek, 1979), les avantages apportés par les technologies mobiles se traduisent par le renforcement de la capacité d'apprentissage des professionnels en entreprise, ce qui est déterminant pour le développement de leur propre professionnalisme (Lui et al, 2003 ; Hall, 1968). En d'autres termes, les technologies mobiles permettent de faire évoluer la situation de travail de ces professionnels d'une situation passive à une situation active pour reprendre les termes du modèle "exigence-contrôle" (Karasek, 1979). 
Les résultats de cette recherche montrent que le renforcement des capacités d'apprentissage se traduit en effet pour les répondants par les possibilités nouvelles offertes par ces technologies dans le domaine de la communication et la coordination des activités n'importe où, n'importe quand :

"L'avantage qu'on peut en retirer c'est peut-être que quand vous allez rentrer les informations, elles sont dedans et si vous avez besoin de les retrouver vous pouvez les retrouver. Vous n'avez pas besoin à chaque fois de reprendre le dossier et de regarder tout ce qu'a eu le patient et tout ça...". (Technicien terrain, E1)

"Ces outils donnent une grosse partie d'informations dont j'ai besoin pour pouvoir négocier avec le client, ça permet et on peut s'en servir comme support audiovisuel, et ça permet de nous donner des éléments pour préparer la visite également, par ex, les C.A. les parts de marché, tous les éléments produits, les argumentaires produits". (Commercial, E8)

"Ce sont des moyens de communication privilégiés. J'ai appris à m'en servir de ces outils là et c'est vraiment... pour moi c'est essentiel." (Collaborateur terrain, E10)

"Ces technologies permettent de rester en contact et de faciliter l'accès à l'information. Le fait d'avoir un ordinateur portable ou un téléphone portable, permet de transmettre des informations beaucoup plus facilement et de recevoir de l'information" (Commercial E10)

Une autre dimension importante, en termes d'apprentissage, est celle de la flexibilité nouvelle que permet l'usage de ces technologies dans l'organisation du travail dans le temps et l'espace pour ces professionnels, à ce titre les estiment mieux contrôler leurs activités grâce à ces outils. Les répondants valorisent particulièrement cette réduction des contraintes en augmentant sensiblement leur autonomie ce qui leur permet d'être dans une situation de travail beaucoup plus active (au sens de Karasek, 1979) :

"le fait que ça me donne une indépendance, que ça permet de travailler de chez moi, le niveau de stress est moindre. Quand je suis au bureau dans un pool, ce que j'ai connu, ò̀ il y a un commercial derrière chaque téléphone, comme j'ai les cordes vocales imposantes, je vais peut-être gêner mon voisin. Alors que là je suis tranquille chez moi, j'ai de meilleures conditions de travail. Je suis satisfait." (Commercial E2)

"Quand on n'est pas sur un client, on peut rester chez soi. Ca m'est arrivé la semaine dernière, j'avais quelques points à clôturer sur un autre dossier, dans ce cas là tu travailles depuis chez toi. Sinon je travaille soit ici, soit chez le client, mais plutôt chez le client. De janvier à mars je passe 3 jours ici au bureau" (Collaborateur terrain, E9).

"Quand on est à l'hôtel le soir et qu'on a le temps de préparer les $r v d u$ lendemain, l'ordinateur évite d'avoir à se préparer 3 ou 4 jours à l'avance, la veille au soir si on a une réunion le lendemain, on peut faire le nécessaire" (Commercial, E9)

"On a une flexibilité qui est réellement appréciable quand on a plein de dossiers qui vous tombent dessus en même temps on réussit à prendre le temps nécessaire pour faire les choses correctement" (Collaborateur terrain, E10)

Au delà de la flexibilité nouvelle permise par ces technologies, les répondants soulignent que ces technologies permettent d'enrichir le situation de travail en développant leur efficacité 
personnelle et en renforçant leurs compétences notamment vis-à-vis du client et ceci d'autant plus que la culture de l'organisation est favorable. Ces perceptions de l'évolution de la situation de la travail sont cohérentes avec le renforcement du contrôle de leurs activités dans le modèle "exigence-contrôle" (Van der Doef \& Maes, 1999 ; Karasek \& Theroell, 1990; Karasek, 1979) :

"Dans un jargon, je dirais que ça à de la gueule! Quand on arrive chez un client avec un ordinateur portable, qu'on lui fait une simulation sur un logiciel, c'est plus officiel, ça met plus en confiance le client. C'est plus crédible que si je lui fait une présentation papier. C'est plus crédible aussi pour présenter les caractéristiques techniques d'un produit, pour avoir la confiance des clients, c'est beaucoup plus facile." (Commercial, E2)

"Oui, gain de temps, plus efficace quelque part. ça améliore la performance. Ça évite de stocker des tas de classeurs. Quand on voyage plutôt que d'avoir le dossier ... on a tout dans l'ordinateur." (Commercial, E3)

"Il ne faut pas que ce soit un blocage vis-à-vis du client parce qu'on se sert de l'informatique pour lui faire une proposition. Au contraire, la proposition est plus carrée, c'est plus professionnel et ça va dans le sens du service client, et à l'extrême de la fidélisation du client"(Resp. opérationnel, E5)

"Pour le reste, je crois que c'est un atout supplémentaire. L'offre est plus professionnelle, on peut imprimer des choses très propres et très nettes pour les vendeurs qui écrivent souvent très mal, c'est un outil bien pratique. On peut proposer des photos au client s'il n'est pas décidé, on a un tas d'offres et de panels qui peuvent être immédiatement mis en action, et ça c'est important. Donc c'est un aspect plus professionnel pour le client qu'on peut avoir face à soi, ça c'est vrai." (Commercial, E6)

L'ensemble de ces perceptions confirme la validité de la deuxième proposition de recherche, traitant des opportunités d'apprentissage, qui constitue l'une des hypothèses fondamentales du modèle "exigence-contrôle". Au-delà de ces conséquences indéniablement positives, il est nécessaire maintenant d'examiner les effets perçus négatifs de ces technologies sur la situation de travail de ces professionnels.

\subsection{Technologies mobiles et situations de travail : un renforcement des contraintes pour les professionnels en entreprise}

Même si les technologies mobiles semblent naturellement s'imposer aux yeux de nombreux répondants, un certain nombre d'entre eux questionne la pertinence du développement de ces outils. Ils n'hésitent pas à remettre en cause la croyance de l'augmentation de l'efficacité individuelle et collective due à l'introduction de ces technologies :

"Les difficultés que l'on rencontre, c'est quand ils changent... Avec l'informatique, vous pouvez aller un peu n'importe où... suivant ce que vous avez à faire donc vous êtes obligé de cliquer dessus suivant ce que vous voulez faire. Mais tout ça, ils réorganisent de temps en temps. Ils réorganisent sans nous prévenir ... ça nuit à l'efficacité du service" (Collaborateur terrain, E1)

"On gagne apparemment du temps, mais on en perd aussi parce qu'on communique beaucoup mais pas forcément sur les choses qui sont importantes." (Collaborateur terrain, E4) 
"Le fait d'être en prise directe, en permanence sur l'information fait que on a tendance à faire circuler de l'information brute de plus en plus rapidement et en quantité de plus en plus importante et on pratique de moins en moins la synthèse. Or, ce qui a de la valeur c'est l'information synthétisée confrontée à d'autres choses. Ca c'est un vrai problème, et à vouloir traiter tout ce qui se passe à chaud, le plus vite possible, et dans les conditions du direct finissent par faire des erreurs graves"(Resp opérationnel, E5).

"Non, je ne pense pas que nous soyons plus performants. L'entreprise nous a fourni ça peut être pour cette raison là mais il y a aussi un effet mode. Tout commercial se doit d'avoir son téléphone portable, son micro portable, son Palm... Utile ou pas utile, ça fait bien de l'avoir, un petit peu gadget quand même." (Commercial, E6)

"Ca peut distraire en réunion. Les réunions sont faites pour ne pas perdre de temps et si on rate une partie parce qu'on a des mails tout le temps... c'est pas rentable.." (Resp opérationnel, E8)

Si le renforcement de l'efficacité grâce à ces technologies est remis en cause par certains, plus nombreux sont ceux parmi ces professionnels qui expriment des réserves quant à l'augmentation de l'exigence dans la situation de travail largement imputable, selon eux, à l'utilisation de ces outils. Si on se réfère au modèle "exigence-contrôle"( Karasek \& Theroell, 1990 ; Karasek, 1979), ils expriment un renforcement sensible de la contrainte dans la situation perçue de travail.

"Ca me stresse oui. Ca le fait d'avoir le téléphone dans la poche tout le temps, oui c'est stressant. D'un côté c'est bien parce qu'on est joignable rapidement, mais d'un autre côté c'est quand même assez stressant.." (Resp opérationnel, E1)

"On confond l'urgent et l'important. Tout est urgent, mais n'est pas forcement important, mais on a oublié ça. Et donc on a ce besoin de communiquer, communiquer..."(Collaborateur terrain, E4)

"Oui les journées sont plus élastiques qu'avant. Ça a été tout à fait un gain et un bénéfice pour l'entreprise. Pour nous j'estime que c'est une perte si on est consciencieux dans son travail. On a tendance avec ces outils à déraper" (Commercial, E6)

"Il faut pas vouloir tout faire, du commercial, de la facturation, du marketing...quand on en fait trop, à un moment, il y a des soucis. Mais bon, ça va évoluer. A la base, il y a quelque chose pour les commerciaux qui n'est pas du tout pratique. C'est vrai que c'est une source d'énervement phénoménale, une perte de temps et une source de stress.." (Commercial, E7)

"D'être sans arrêt dérangé, etc.., je pense que oui, oui je pense que, je dirais de façon effectivement caricaturale et exagérée on est tous sur le qui-vive. Il y a sans arrêt, sans arrêt, sans arrêt, des offres à répondre, des demandes qui nous arrivent et là c'est pareil, il faut apprendre à répondre rapidement, bien connaître les procédures pour aller plus vite.." (Resp. opérationnel, E9)

"Je dirais aussi qu'il y a de l'abus dans le sens où nous, de plus en plus on nous envoie des mails, il y a une circulation de l'information qui est très top-down, c'est-à-dire que ça permet aux gens de bombarder de mails, de messages, de choses comme ça, sur les chefs de secteur qui sont en fait la seule porte vers le 
terrain, et vers les clients; et trop d'information tue l'information ce qui fait que recevoir de 50 à 60 mails par jour, sachant qu'on ne les regarde que le soir, entre 6 heures et 8 heures en général, voire plus tard..." (Commercial, E10)

Enfin, toujours sur la perception de l'augmentation de la contrainte dans la situation de travail, c'est l'impact de ces technologies sur la sphère hors travail qui est perçu comme renforçant encore l'exigence tout en réduisant les capacités de contrôle des activités (latitude des décisions), c'est-à-dire l'autonomie recherchée et valorisée pourtant par ces professionnels en entreprise. Cette situation correspond explicitement à la situation de très forte contrainte décrite dans le modèle "exigence-contrôle" (Karasek, 1979) :

"J'essaie de leur laisser peu la place... Moi je ne prends dans ces technologies que le plus, mais j'excède... Donc en clair je me protège par rapport au fait qu'elles ne m'envahissent pas trop. C'est ça l'idée qu'il y a derrière: utile mais pas envahissante." (Resp. opérationnel, E1)

"Il est important pour certains d'envoyer les e-mails, de répondre aux e-mails, de créer des volumes de manière fictive. Nos collègues de New York, qui ont les Blackberrys, c'est vrai que ça nous fait toujours rigoler quant on voit que les gens ont envoyé leurs mails à $6 \mathrm{~h}$ du matin. Quelqu'un qui prend son petit déjeuner le matin ne lit pas son journal, il répond à ses mails. C'est pareil avec le téléphone portable..." (Resp opérationnel, E4)

"Ca dépend. L'objectif est de ne pas travailler pendant les vacances, mais la réalité est parfois différente. Si on a un projet quand on est en vacances, on doit le suivre. Et c'est vrai que je pars en vacances avec mon ordinateur. Je me branche sur le téléphone..." (Collaborateur terrain, E4)

"L'inconvénient le plus net, le plus marquant de ça, c'est qu'on gomme de plus en plus la frontière entre ce qui est la vie professionnelle et ce qui est la vie personnelle. Il y a 30 ans, quand j'étais au bureau, j'étais au travail, quand je n'étais pas au bureau, je n'étais pas au travail. Aujourd'hui, si j'ai quelque chose d'urgent à faire, etc. Je sais le faire le week-end depuis chez moi. Et comment j'arrive à gérer ça? Est-ce que je dois m'occuper de mes enfants, ou est-ce que je dois travailler le week-end?" (Resp. opérationnel E5)

Les perceptions recueillies sur les effets négatifs des technologies mobiles ne sont ici que partiellement reproduites, ce qui traduit une inquiétude sérieuse de ces professionnels quant au renforcement de la contrainte dans les situations de travail utilisant largement ces technologies comme le suggère la troisième proposition de recherche. Si les professionnels en entreprise perçoivent une augmentation de l'exigence et parallèlement une diminution de leur autonomie, il convient de réfléchir à un renforcement du soutien social pour limiter les conséquences négatives de l'usage de ces technologies comme le suggère le modèle enrichi "exigence-contrôle-soutien social" proposé par Johnson \& Hall (1988). C'est à la construction de ce soutien social et plus généralement à la diminution de la contrainte dans la situation de travail des professionnels en entreprise que sont appelés les managers de ces professionnels dans les politiques et pratiques qu'ils mettent en œuvre. 


\section{Conclusion : Le management des professionnels en entreprise, limites et pistes futures de recherche}

Cette première recherche exploratoire sur les professionnels en entreprise met en évidence la pertinence du modèle " exigence-contrôle » pour appréhender les effets de la recomposition actuelle des environnements de travail liée à l'introduction des technologies mobiles.

Comme toute recherche exploratoire, les limites de cette recherche tiennent largement à son à l'échantillon des entreprises retenues et des répondants. La généralisation de résultats nécessite, dans le cadre de recherches futures quantitatives, d'inclure une diversité beaucoup plus grande des entreprises concernées par le développement des technologies mobiles mais également des individus et notamment ceux qui utilisent peu ou pas ces technologies. Les recherches futures devront également inclure des catégories de professionnels de type indépedants afin de préciser si les nouvelles situations de travail mobiles sont perçus de façon différentes selon que les professionnels s'appuient sur une structure d'entreprise ou non. D'autres pistes de recherche future peuvent concerner les questions de transformation des relations de pouvoir au sein des organisations en s'appuyant sur les travaux des sociologues de l'organisation (Crozier \& Friedberg, 1977). La transformation des identités professionnelles constitue également une voie de recherche intéressante: comment l'identité des professionnels nomades se constitue-t-elle?

L'adoption et le développement de l'usage des technologies mobiles transforment assez sensiblement les situations de travail des professionnels en entreprise en abolissant notamment les frontières du temps et de l'espace. Les conséquences paradoxales de l'usage de ces technologies mises en évidence par le résultat de l'étude menée dans dix organisations françaises interpellent le management de ces professionnels dans la mesure où leur situation de travail est susceptible d'être assez profondément recomposée avec le risque d'une perte progressive d'une identité professionnelle largement fondée sur l'autonomie. Pour éviter ce risque, les managers responsables de ces professionnels en entreprise doivent créer les conditions du développement de compétences nouvelles en s'appuyant les opportunités nouvelles d'apprentissage permises par ces technologies.

\section{Références}

Arnold M. (2003), "On the phenomenology of technology: the "Janus-faces" of mobile phones", Information and Organization, 13, p. 231-256

Aubert N., (2003), : Le culte de l'urgence. La société malade du temps, Paris, Flammarion

Aubert N., Gruère J.P, Jabes J, Laroche H.,\& Michel S. (1991) : Management, aspects humains et organisationnels, Paris, PUF Fondamental.

Besseyre des Horts; C.H (2004) : "La fonction R.H. doit-elle mesurer le retour sur investissement R.H pour être stratégique ?', Montréal, Actes du $15^{\text {ème }}$ congrès de l'AGRH, p.185-208.

Chen L. \& Nath R. (2005) : "Nomadic culture : cultural support for working anytime, anywhere", Information Systems Management, Fall 2005, p. 56-64 
Churchill E.F., Munro A.J. (2001), "Work/place: Mobile technologies and arenas of activity", ACM SIGGROUP Bulletin, Vol. 22, n 3, p. 3-9

Claessens B.J., Van Eerde W.E., Rutte C.G., \& Roe R. (2004) : "Planning behavior and perceived control of time at work", Journal of Organizational Behavior, vol 25, p.935-950

Cocula F. et Fredy-Planchot A "Pratiquer le management à distance", Gestion 2000, n¹, janvier-février 2003, pp.43-63.

Cousins C.K.and Robey D. (2005), "Human agency in a wireless world: Patterns of technology use in nomadic computing environments", Information and Organization, Vol. 15, $\mathrm{n}^{\circ} 2$, p. $151-180$

Crozier M. \& Friedberg E. (1977) : L'acteur et le système, Paris, Le Seuil.

Davis G.B. (2002) : "Anytime/anyplace computing and the future of knowledge work", Communications of the ACM, $\mathrm{Vol} 45, \mathrm{n}^{\circ} 12, \mathrm{p} .67-73$

Ettighoffer D. (2001), L'entreprise virtuelle, Nouveaux modes de travail, nouveaux modes de vie?, Paris, Editions d'Organisation

Gribbins M.L., Gebbauer J., Shaw M.J. (2003), "Wireless B2B mobile commerce: a study on the usability, acceptance, and process fit", Ninth Americas Conference on Information Systems

Isaac H., (2006), “Le manager à distance: l'émergence d'un manager nomade”, in Kalika Ed., "Management \& TIC", Editions Liaisons, octobre, 256 p., Paris

Isaac H., Kalika, Campoy E., (2006), "Contribution des technologies de l'information à la perception de l'urgence et de la surcharge informationnelle chez les salariés français : une analyse longitudinale », Communication Colloque « e-GRH », AIM-AGRH, Université Paris Dauphine, Mai.

Isaac H. (2004), "Technologies mobiles et management: l'émergence d'un manager nomade?", gème Congrès de l'AIM

Isaac H. \& Kalika M. (2001), "Organisation, technologie de l'information et vie privée", Revue Française de Gestion, n ${ }^{\circ}$ 134, p.

Jarvenpaa S.L \& Lang K.L (2005) : "Managing the paradoxes of mobile technology", Information Systems Management, Fall 2005, p. 7-23.

Johnson J.V. \& Hall E.M. (1988) : "Job strain, workplace social support, and cardiovascular disease : a cross-sectional study of a random sample of the Swedish working population", American Journal of Public Health (AJPH), Vol 78, 10, p. 1336-1342.

Karasek R.A (1979) : "Job demands, job decision latitude, and mental strain : implications for research design", Administrative Science Quarterly, Vol 24, p. 285-311 
Karasek R.A \& Theorell T.(1990) : Healthy work : Stress, Productivity and the Reconstruction of Working Life, New York, Basic Books.

Leclercq A. \& Isaac H. (2005) : " l'adoption des technologies mobiles par les managers : pour un dépassement des modèles TAM", 10e congrès de l'AIM. , 12p.

Leung K. \& Antypas J. (2001), "Improving returns on M-commerce investments", Journal of Business Strategy, Vol. 22, n 5, pp.12-1

Livian Y.F. (1995) : Introduction à l'analyse des organisations, Paris, Economica, Collection Gestion Poche

Lyytinen K. \& Yoo Y. (2002a) : "Issues and Challenges in ubiquitous computing", Communications of the ACM, Vol 45, $\mathrm{n}^{\circ} 12, \mathrm{p} .63-65$

Lyytinen K., Yoo Y. (2002b), "Research Commentary: the next wave of nomadic computing", Information Systems Research, Vol. 13, n 4, pp. 377-388

Robey D., Lyytinen K., Varshney U., Davis G., Ackerman M.S., Avital M., Sawyer S., Sorensen C. (2004), "Surfing the next wave: design and implementation challenges of ubiquitous computing environments", Communications of the Association for Information Systems, Vol. 13, pp. 697-716

Rodriguez I., Jesus Bravo M., Peiro J.M., \& Schaufali W. (2001) : "The demands-controlsupport model, locus of control and job dissatisfaction : a longitudinal study, Work \& Stress, Vol 15, 2, p.97-114.

Rojot J. (2003) : Théories des organisations, Paris, Editions Eska

Schein E. (1984) : Organizational culture and leadership, San Francisco, Jossey Bass

Ulrich D. (1997) : Human resources champions, Boston, Harvard University Press

Van der Doef M. \& Maes S. (1999) : "The job demand-control (-support) model and psychological well-being : a review of 20 years of empirical research", Work \& Stress, Vol 13,2, p.87-114.

Van Yperen N. \& Hagedoorn M. (2003) : "Do high job demands increase intrinsic motivation or fatigue or both ? the role of job control and job social support", Academy of Management Journal, Vol 46, 3, p. 339-348.

Varshney U. (2003), "Mobile and wireless information systems: applications, networks, and research problems", Communications of the Association for Information Systems, Vol. 12, Article 1

Wiberg M.\& Grönlund A. (1999), "Mobility in the wild, Social aspects of mobile work: A framework for future research and design", Proceedings of the 1999 AMCIS Conference 


\section{Annexe}

\section{Guide d'entretien (collaborateur de terrain)}

\section{Question introductive :}

Pouvez-vous vous présenter (expérience, ancienneté, position hiérarchique, activité) et me décrire quelles sont vos tâches quotidiennes ?

Questions de base sur les technologies mobiles :

1. Pouvez-vous me dire quelles sont les technologies mobiles que vous utilisez dans votre activité professionnelle?

2. À qui appartiennent ces technologies, à vous-même ou à l'entreprise ?

3. Quels sont les usages de ces technologies mobiles dans vos activités quotidiennes ?

4. A quelle fréquence utilisez-vous ces technologies ?

5. Depuis quand êtes-vous équipé de ces technologies?

6. Quelles sont les raisons, selon vous, pour lesquelles votre entreprise vous a équipé de technologies mobiles?

7. Avez-vous éprouvé certaines réticences à utiliser ces technologies?

\section{Questions de relance :}

1. Avez-vous ressenti une certaine pression à utiliser ces technologies de la part de votre hiérarchie?

2. Vos collaborateurs utilisent-ils des technologies mobiles dans leurs tâches quotidiennes ? Vous ont-ils poussé à adopter ces technologies?

3. Ces technologies ont-elles modifié l'organisation de votre temps de travail ?

4. Etes-vous amené à utiliser ces technologies en-dehors du temps de travail ?

5. Etes-vous amené à prendre des décisions en dehors de l'entreprise ? En dehors de vos horaires de travail ? si oui de quelle nature ?

6. Ces technologies ont-elles modifié l'organisation de votre lieu de travail ?

7. L'utilisation de ces technologies a-t-elle modifié les relations avec vos collègues dans votre équipe ? et vis-à-vis des autres salariés de l'entreprise?

8. L'utilisation de ces technologies a-t-elle modifié l'évaluation de votre travail par votre hiérarchie ? le contrôle de vos activités a-t'il été renforcé ?

9. Ces technologies ont-elles une influence sur votre vie privée ?

10. Ces technologies ont-elles une influence sur votre état de santé ou de stress ?

11. Quels principaux avantages retirez-vous de l'utilisation de ces technologies?

12. Aviez-vous conscience de ces bénéfices éventuels avant d'adopter ces technologies?

13. Estimez-vous que l'utilisation des technologies mobiles améliore votre performance dans vos activités professionnelles?

14. L'utilisation de ces technologies a-t-elle modifié votre façon de participer dans votre équipe ? Comment?

15. Pensez-vous que la bonne utilisation des technologies nomades peut substituer les réunions de l'équipe dans votre métier?

16. Ont-elles modifié vos relations avec les clients ? Ont-elles modifié la façon dont vous êtes perçu par vos interlocuteurs (clients, fournisseurs)?

17. Eprouvez-vous un sentiment de satisfaction, de plaisir ou de distraction lorsque vous utilisez ces technologies?

18. Quelles sont les difficultés que vous rencontrez dans l'usage de ces technologies dans vos activités quotidiennes? 
19. Vous sentez-vous encadré et soutenu lors de l'utilisation de ces technologies, notamment en cas de problème et de panne ?

20. Avez-vous été formé à l'utilisation de ces technologies? 